Results. The results of this form of treatment are already available in the two papers I have quoted. Follow-up of these cases is at present being prepared but unfortunately it is not yet available.

The published figures show that the percentage of patients discharged with sterile urine is higher than has been described following any other method of initial treatment. Whilst appreciating that intermittent catheterisation properly carried out makes serious inroads on the working time of medical officers, I feel that from the patient's point of view the end more than justifies the means.

\title{
REFERENCES
}

Guttmann, L. (1947). Proc. Roy. Soc. Med. 40, 219.

Guttmann, L. (1949). Br. Surg. Pract. 6, 445.

Guttmann, L. \& Frankel, H. (I966). Int. F. Paraplegia, 4, 63.

Lapides, T., Ajemian, E. \& Lichtward, T. (I960). f. Urol. 84, 609.

\section{NEUROGENIC BLADDER: CONSERVATIVE METHODS OF TREATMENT}

\author{
By S. A. VinCENT \\ Belfast City Hospital
}

Introduction. The treatment of the symptom of urinary incontinence from any cause is much influenced by the fact that, although the patient may complain only of incontinence of a varying degree, it can be found on investigation that obstruction is present as well.

This occurs even in bed-wetting children who have got frequency and urgency by day and in adult patients with exactly similar diurnal symptoms, the difference between urgency and urge-incontinence being only one of degree.

In bladders that have been deprived in whole or in part of their nerve supply the importance of the obstructive element has been emphasised by many workers, and methods of eliminating it have been described. Ross, Damanski and Gibbon (1967) have reported on a series of neurogenic bladders where division of the external sphincter has been successful in a high proportion of cases in relieving the obstructive element.

One of the conservative methods of treatment to be described depends on deliberately obstructing the flow of urine, and in using this method any obstructive effect already present must be treated with the greatest respect, whether in a neurogenic bladder or not. If it is of a degree sufficient to endanger renal function, it must form an absolute contra-indication to 'artificial mechanical obstruction' as a method of treatment.

Fortunately, the obstructive effects are not by any means proportional to the degree of incontinence. Thus, a patient may have only slight urgency and yet cystography may show increased bladder trabeculation, diverticula formation, reflux into dilated ureters, hydronephrosis and pyelonephritis. Chemical tests may show grossly impaired renal function. On the other hand, the incontinence may be very severe and investigation may fail to reveal any obstructive element, 
so that in these cases artificial mechanical obstruction to the flow of urine may be attempted even in neurogenic bladders.

Effect of Perineal Elevation. When the ischiorectal region and perineum is elevated beyond a certain point by any mechanical means, the flow of urine is stopped or prevented from starting, and in certain patients with gross urgency the urgency ceases suddenly when the elevation has reached a certain point.

As examples of patients making this observation themselves the writer has a series of I 5 young girls who can stop their urgency and incontinence by sitting firmly on their heel when they feel the urgency starting, and many patients, mainly female, notice that their urgency and incontinence only start when they get up from a chair. Some of these have learned to sit rather far forward on the corner of a chair, which they allow to press upward on the perineum.

That the effect is not produced merely by squeezing the urethra at a low level is indicated by the fact that ciné micturating cystograms show the urethra to close and to leave no X-ray opaque material in it. Squeezing the urethra alone allows the fluid to remain right down to the point of pressure.

Based on these observations, an appliance has been designed which is capable of maintaining an adequate elevation of the perineum without undue discomfort and a simple new method of preliminary investigation has been evolved which can indicate the value or otherwise of a new conservative method of treatment.

These methods will be described in relation to patients with urinary incontinence from all causes before considering the various special problems that arise when they are applied to patients with neurogenic bladders.

Preliminary Investigation. The preliminary investigation is performed in the out-patient department at the first visit. Many of the patients complain of frequency and urgency of a varying degree. When the urgency is at its height and incontinence is imminent the perineal region is elevated by pressing it firmly upwards manually. In a proportion of patients this manipulation relieves the urgency, which returns on removal of the elevation. The test is then repeated several times until it is made clear to the patient that the lifting is stopping the urgency.

In male patients the manipulation is used to show the patient that it stops the actual flow of urine, or prevents it from starting without discomfort; in female patients with stress incontinence it is used to demonstrate that with the pressure applied urine does not leak away on coughing.

If the test is positive (and in almost half of all patients with urgency this is so), the patient is seated on a firm chair with a large pad of cotton-wool under the perineum. The time that elapses between the seating of the patient and the return of the urgency gives a fair indication as to the value of the future conservative treatment. If it is only five or ten minutes, the chances of improving the patient are generally not great. Anything over half an hour indicates a fair prospect of success.

After a varying time the desire to pass urine returns, either in the form of the original urgency or (in the successful cases) in the form of a normal desire to empty a normally full bladder. The patient is then given a bottle or bed pan and the amount of urine passed is measured. In some cases this may be only several ounces but in others it is many times the usual voiding volume. 
Conservative Treatment. In the latter event it may be decided to give the patient an appliance to keep the pelvic floor elevated or to allow the patient to use the 'pad and chair' method, but in either case the patient is also instructed in a simple exercise designed to strengthen the pelvic floor muscles, or at least to bring them more under the control of the mind. The exercises consist of tightening the anus and the buttocks as powerfully as possible, and this may indeed be the sole method of treatment; that is, no pad or applicance is used. The exercises are repeated many times through the day, both sitting and standing. If they prove successful no further treatment is necessary, but sometimes the patient must be referred to the physiotherapy department for supervision of the exercises and for a six-week course of faradism to the perineum and anal regions.

The physiotherapists do this by using one large saline-soaked pad electrode over the sacrum and another covering a large area of the perineal region. It is important to request the physiotherapist to make the perineal electrode the smaller and to observe the actual contraction of the anus and perineal muscles with each surge of faradism. Where possible she should observe this stimulation stopping the flow of urine on one or more occasions.

In certain cases, particularly in patients with mechanical weakness of the pelvic floor, these methods may be successful by themselves; but in others the patient is only controlled by using the appliance in the form of a wide belt which holds a saddle piece, with a balloon on its upper surface, tightly pressed against the anus and perineum (Vincent, 1959, 1960, 1964, I966a, b).

Applications in Neurogenic Bladders. When this method of investigation and treatment is applied to patients with neurogenic bladders, many factors are present that render the patient unlikely to receive spectacular help.

In upper motor neurone lesions the bladder, like the patient, is spastic to a varying degree and the completely random waves of contraction may be capable of overcoming any acceptable amount of perineal elevation. With complete lesions associated with anaesthesia the danger of pressure sores generally precludes the use of the amount of elevation necessary to keep the bladder outlet closed, and when the obstructive effects of the condition are marked the method is contraindicated.

On the other hand, when the lesion is incomplete and the patient ambulant, particularly in the static or very slowly developing type of multiple sclerosis (where the afferent nerves are not affected), a small proportion of patients have been completely relieved of their symptoms in a few weeks and are still symptom-free several years later. It is not possible to predict which patient will benefit, but the simple essential tests in the out-patients department form a reasonable guide as to the value of the method.

In cauda equina lesions the amount of pressure required to maintain an elevation sufficient to keep the bladder from leaking is usually much less, since the muscles are relaxed and, although many patients have large areas of perineal anaesthesia, no pressure sore has been caused by these methods. Against the obvious advantage of the smaller pressure requirements must be placed the observation that many of these patients have got ureteric reflux and dilated ureters, so that any obstructive effect is transmitted directly to the kidneys. On the other hand, the pressures in the dilated paralysed ureters are not great and some of these patients found considerable benefit from the perineal elevating appliance. If there 
is complete paralysis of the anus and pelvic floor, perineal exercises are, of course, not possible and faradism is no help. Although a number of meningo-myelocele children and young adults have been helped by the appliance and have been kept quite dry, in the long run many of them have finally required some form of diversion operation after a period varying from several weeks to several years, when it became apparent that the renal function was at some risk. As with upper motor neurone conditions the main value is in incomplete lesions.

Infection. The above method of treatment has not, in the past, been accompanied by any deliberate study of the pattern of urinary infection in relation to the neurogenic bladder or to the many difficult bladder neck conditions associated with urinary incontinence. With the improved routine methods of taking urine samples, prostatic massage, bacterial counts and vestibular swabbing introduced by Stamey (1968), an investigation has been started on a large series of patients with urinary incontinence from all causes in an attempt to clarify the relationship between the infective and mechanical factors involved in abnormal bladder control.

Alleviation. In many patients with urinary incontinence due to the neurogenic bladder or other cause the above methods are not feasible or have proved unsuccessful. It is not proposed to discuss the undoubted value of the various operations or methods of catheterisation in these cases and this communication will be confined to those cases where it has been decided that all that remains is to allow the urine to escape from the urethra.

In the male it is possible to collect the urine as it escapes, but in the female it is almost taken for granted that the urine must be allowed to be absorbed as far as possible by incontinence pads. This, in effect, means that the patient is wet for a variable time and frequently a high proportion of the day and night.

The incontinence pads may be 'loaded' with a powder similar in nature to the well known 'Polycell' powder, ${ }^{1}$ a method evolved in Leeds and in Pinderfields Hospital, Wakefield, by Phillip Smith et al. The urine is rapidly absorbed by the powder and forms a gel which is not wet to the touch and from which the urine cannot be squeezed again. This method deserves further study and development as it can be used even in bed-fast patients who are quite unco-operative and who actively resist all attempts to keep them dry by appliances.

For female patients, however co-operative, it is generally agreed that no efficient incontinence appliance is available commercially.

This opinion was confirmed by purchasing all the appliances available and testing them on female volunteers from a general surgical ward. These patients had no urinary complaint and were able to pass urine at will with the appliances very carefully adjusted according to the makers' instructions. In no case did any appliance collect more than a small proportion of the urine in the container, and this lack of success was found to be due to certain faults which the writer has spent a number of years attempting to correct.

Various shapes and consistencies of plastic appliances were fashioned in a workshop in the Anatomy Department of Queen's University, Belfast, and a technique was evolved which enabled the appliances to be turned out in a short

1 Methyl cellulose ('celacol') obtainable from J. M. Steel \& Co. Ltd., I44 St Vincent Street, Glasgow, C.2. 
time in sufficient numbers for clinical trial and modification where it was found necessary to eliminate any faults that had been discovered.

As a result of this method of trial and error an appliance has been evolved which is capable of collecting all the urine by day and by night in cases of spinal injury, multiple sclerosis and bladder outlet insufficiency from other causes. Close co-operation and enthusiasm on the part of the patient and her attendants are necessary in the early stages. The appliance consists of a soft pneumatic P.V.C. vulvar attachment which is prevented from the lateral compression of the patient's thighs by providing a base of more resilient P.V.C. A large tube hinges on the posterior part of this base and cannot kink. The tube contains a wide 'fish-tail' valve which is enclosed in rigid tubing to prevent compression, and this is invaginated into a boat-shaped container which extends forward and backwards from the patient's vulva. By extending it in a sagittal direction it is possible to confine the vertical distance from the patient's vulva to under 4 inches and so avoid pressure on all the container at once, as can occur with the convertible bag which also extends much lower down.

The importance of the container extending only a short distance below the vulva lies in the fact that with the modern hem-line, some patients, both walking and chair-fast, stated that greater distances were quite unacceptable and this was taken as an excellent sign not only of the presence of the will to live but also of the will to 'live it up'. With the newer containers this type of patient co-operated with enthusiasm.

The vulvar attachment with various forms of container has been used constantly on several patients for over two years and the device in its present form is in use on these patients and on five more. Three of these have multiple sclerosis, one has a high spinal injury and the others have incontinence which has not been cured by many gynaecological operations.

These patients find it to be quite acceptable and it is capable of keeping them dry by day and by night. A number of patients have stopped using it, usually after an insufficient trial, and in some instances its use has beendiscontinued as it was evident that the patient or her attendants preferred to revert to incontinence pads. Those who persisted for several weeks and who had marked success with it during that time have found it to be of help. In its present form the apparatus appears to mark a stage in our search for a really satisfactory female incontinence appliance.

In six patients (two with multiple sclerosis), it was possible to discontinue its use, as the patient had become much more continent or even quite dry. This was considered to be due to the perineal elevation effect of the posterior part of the vulvar attachment, a very satisfactory and unexpected result. In many other cases it was rather a disadvantage as it added an undeniable obstructive effect to a very variable degree. Thus some patients were unable to pass urine with it on.

If any obstructive element with renal risk was present its use was discontinued. The more incontinent the patient, the greater the chance of success with the appliance.

Conclusion. In urinary incontinence, as in so many other conditions, conservative methods can involve more work and more worry than operations, but they are often justified. These methods are presented as a further method of study of bladder function with possibilities of alleviation and treatment which have not yet been fully explored. 


\title{
REFERENCES
}

Ross, J. Cosbie, Gibbon, N. O. K. \& Damanski, M. (i967). B.f.S. 54, No. 7.

STAMEY, T. (1968). F. Urol. 97, (May).

VINCENT, S. A. (1959). Ulster med. four. 28, 176.

VINCENT, S. A. (I960). Lancet, 2, 292.

Vincent, S. A. (I964). Dev. Med. and Child Neurol. 6, 23.

Vincent, S. A. (I966a). Lancet, Sept., 63I-632.

Vincent, S. A. (1966b). Bio-Engineering, Sept., p. I.

\section{THE ROLE OF THE EXTERNAL SPHINCTER}

\author{
By J. Cosbie Ross \\ Director of Urological Studies, University of Liverpool
}

Introduction. It must be acknowledged that as yet no one knows the precise role of the external sphincter and there should, by right, be a question mark after the word 'sphincter'. The problem is much more complex and obscure than the simple, easily understood mechanism of the anal sphincter.

However, there is much that is already known, and perhaps recent work has shed some light on the problem.

First, the traditional view. In the 32 nd Edition of Gray's Anatomy (I958), the description is as follows. 'The sphincter urethrae surrounds the membranous portion of the urethra, and lies deep to the inferior fascia of the urogenital diaphragm. Its superficial or inferior fibres arise in front from the transverse perineal ligament and from the neighbouring fascia. They pass backwards on each side of the urethra and converge on the perineal body for their insertion. Its deep fibres, some of which arise from the fascial sheath of the pudendal vessels and pass medially, form a continuous circular investment for the membranous urethra.'

Actions. 'The muscles of both sides act together as a sphincter, compressing the membranous part of the urethra. During micturition they, like the bulbocavernosus, are relaxed, and only come into action at the end of the process to eject the last few drops of urine.'

The authors go on to describe the transverse perineal ligament as the thickened apical part of the inferior fascia of the urogenital diaphragm attached laterally to the inferior pubic rami. There is no doubt that this classic description has been modified by the work of recent investigators. Firstly, Bors (1954) has shown many years ago that the original tidy concept of striated muscle fibres being confined between the two layers of the urogenital diaphragm was incorrect as he found striated muscle extending up the prostatic urethra to the bladder neck, being especially marked at the anterior commissure. Confirmation of this was afforded by the fact that during the years of 1952 to 1956 (when the writer carried out the operation of pudendal neurectomy for obstruction at the external sphincter, and before it was realised that division of the muscle was a better procedure) it was found that following the operation the whole posterior urethra frequently opened out and relaxed.

In a histological study Beneventi and Marshall (1956) found that the fibres of the external sphincter blended with the prostatic capsule, extending dorsally 